\title{
Globe Axial Length Growth at Age 5 Years in the Infant Aphakia Treatment Study
}

\author{
M Edward Wilson, MD ${ }^{1}$, Rupal H. Trivedi, MD MSCR ${ }^{1}$, David R. Weakley Jr., MD², George A. \\ Cotsonis, MD $^{3}$, Scott R. Lambert, MD $^{4}$, and for The Infant Aphakia Treatment Study Group* \\ ${ }^{1}$ Storm Eye Institute, Medical University of South Carolina, Charleston, SC \\ ${ }^{2}$ Department of Ophthalmology, Southwestern University, Dallas, TX \\ ${ }^{3}$ Department of Biostatistics and Bioinformatics, Rollins School of Public Health, Emory \\ University, Atlanta, GA \\ ${ }^{4}$ Department of Ophthalmology, Stanford University School of Medicine, Stanford, CA
}

\begin{abstract}
Purpose-To report the longitudinal change in axial length from the time of unilateral cataract surgery at age 1-7 months to age 5 years, and to compare axial length growth of operated eyes to that of fellow unoperated eyes.
\end{abstract}

Design-Comparative case series

Subjects-Infants enrolled in Infant Aphakia Treatment Study

Method-Axial length at baseline and age 5 years and change in axial length were analyzed relative to treated versus fellow eye, visual outcome and treatment modality (contact lens versus IOL). Eyes with glaucoma or glaucoma-suspect were excluded from primary analysis but reported separately

Main outcome measure-Axial length growth from preoperative to age 5 years.

Results-Seventy patients were eligible, however axial length data for both eyes were available for 64 patients at baseline and 69 patients at age 5 years. Axial length was significantly different between treated and fellow eyes, preoperatively $(18.1 \mathrm{~mm}$ versus $18.7 \mathrm{~mm}, \mathrm{p}<0.0001)$, and at the final follow-up ( $21.4 \mathrm{~mm}$ versus $22.1 \mathrm{~mm}, \mathrm{p}=0.0004)$. The difference in AL growth between treated and fellow eyes was not significant $(3.3 \mathrm{~mm}$ versus $3.5 \mathrm{~mm}, \mathrm{p}=0.31)$. The change in AL in eyes was similar with both treatments (contact lens $3.2 \mathrm{~mm}$ and IOL $3.4 \mathrm{~mm}, \mathrm{P}=0.53$ ) and did not correlate with visual outcomes $(\mathrm{P}=0.85)$. Eyes receiving additional surgery to clear the visual axis opacification grew significantly more as compared to eyes not receiving surgery to clear visual axis ( 3.8 versus $2.7 \mathrm{~mm}, \mathrm{P}=0.013$ ). Twenty glaucoma patients and 16 glaucoma-suspect patients were excluded from the above analyses. As a group, glaucoma patients showed

\footnotetext{
Corresponding Author: M. Edward Wilson, MD.

See Appendix for full listing of Infant Aphakia Treatment Study Group

Proprietary interests: none

Trial Registration: clinicaltrials.gov Identifier NCT00212134
} 
significantly more eye growth $(5.7-\mathrm{mm})$ than non-glaucoma patients $(3.3-\mathrm{mm})$ and glaucoma suspect (4.3-mm).

Conclusion-Eyes treated for monocular cataract in infancy have similar axial growth to that of fellow eyes, despite having a shorter AL at the time of surgery. The change in AL in eyes was similar with both treatments (contact lens and IOL), did not correlate with visual outcomes and was higher in eyes receiving additional surgery to clear the visual axis or eyes diagnosed with glaucoma.

\section{Introduction}

The effect of removing the crystalline lens on axial elongation during early childhood is poorly understood. Accurately predicting axial elongation is particularly important when an intraocular lens (IOL) is implanted into a child's eye. If greater axial elongation than anticipated occurs, it may be necessary to exchange the IOL when the child is older. Alternatively, if less axial elongation than anticipated occurs, the child may need to wear spectacles or contact lenses for hyperopic correction on a long-term basis. ${ }^{1}$ Choosing an IOL power for implantation into a child's eye is complicated by continued and unpredictable ocular growth. Understanding globe axial length (AL) growth helps the surgeon to achieve the desired refractive error when the eye is fully grown. The prediction and management of refractive error changes over time is a major challenge for the long-term care of infants operated for cataract surgery. Various factors influence growth of an eye after cataract surgery, including but not limited to, age at cataract development and surgery, visual acuity, and glaucoma. These effects have important clinical implications in determining the optimal IOL power to implant in a child to achieve a preferred refractive correction later in life.

The Infant Aphakia Treatment Study (IATS) is a randomized clinical trial comparing the effect of primary IOL implantation versus aphakia corrected with a contact lens (CL) in infants 1 to 6 months of age following unilateral cataract surgery. AL for each eye at the time of surgery and AL of those eyes at 1 year of age in the IATS have been reported. ${ }^{2}$ At baseline, eyes with cataracts were shorter than fellow eyes. During the first year of life, the change in AL per month was smaller in operated eyes treated with a CL than it was in operated eyes treated with an IOL, and the difference was not significantly related to age at surgery. ${ }^{2}$ Herein, we report globe AL growth at age 5 years of age in the IATS.

\section{Methods}

The study followed the tenets of the Declaration of Helsinki, was approved by the institutional review boards of the participating institutions, and was in compliance with the Health Insurance Portability and Accountability Act. The main inclusion criteria were a visually significant congenital cataract ( $>3 \mathrm{~mm}$ central opacity) in one eye, a normal fellow eye, and an age of 28 days to $<210$ days at the time of cataract surgery. Patients were randomized either to have an IOL placed at the time of the initial surgery or to be left aphakic and optically corrected with a CL. Details of the study design, surgical technique, follow-up schedules, patching and optical correction regimens, evaluation methods, biometry technique, definitions used for glaucoma and glaucoma-suspect, patient characteristics at baseline and the method to define reliable A-scan ultrasound tracings have 
been reported previously. ${ }^{2,3}$ Measurements from invalid, unreadable, and missing A-scan ultrasound tracings were not included in the analyses. A-scan tracings from patients with glaucoma or glaucoma suspect were also excluded from the primary analysis because of the excessive axial elongation that can occur in infantile eyes with glaucoma. This group was analyzed separately.

We analyzed AL at baseline and at age 5 years. We calculated the change in AL and analyzed that change relative to treated versus fellow eyes, treatment modality (contact lens versus IOL), the presence or absence of additional intraocular surgery to clear visual axis, and visual outcome. For visual outcome, eyes were divided into four categories based on visual acuity at age 4.5 years: $20 / 20$ to $<20 / 40,20 / 40$ to $<20 / 80,20 / 80$ to $<20 / 200$ and $20 / 200$ or worse.

The axial length of operated eyes was compared to that of fellow eyes using a paired t-test. Independent group t-test was used to compare data from the CL and IOL groups. One-way analysis of variance was used to compare $\mathrm{AL}$ at age 5 years in the treated eye according to the visual acuity outcome category. For all analyses, a $\mathrm{P}$ value $<0.05$ was deemed statistically significant. All statistical analyses were done with SAS 9.2 (SAS Institute Inc., Cary, NC).

\section{Results}

\section{Patients}

Of the 114 patients enrolled (57 per treatment), one patient in the IOL group was lost to follow-up at age 18 months. The remaining 113 patients all had a clinical examination at age 5 years (mean, 5.0 years; range, $4.7-5.4$ years) with an average length of follow-up of 4.8 years (range, $4.4-5.3$ years) after surgery. Twenty glaucoma patients (contact lens group = $9, \mathrm{IOL}=11$ ) and 16 glaucoma-suspect patients (contact lens group $=11, \mathrm{IOL}=5$ ) were excluded from primary analyses. From the remaining patients, valid AL data of both eyes were available for 64 patients at baseline and 69 patients at age 5 years. Of this, 56 patients have both preoperative and 5-year AL data available. Table 1 and e-supplement figure 1 illustrates $\mathrm{AL}$ in treated and fellow eyes at baseline and age 5 years. There was no significant difference between the AL growth of treated eyes and fellow eyes $(0.2 \mathrm{~mm}, \mathrm{P}=$ 0.31 table 1). Table 2 and e-supplement figure 2 illustrate $\mathrm{AL}$ growth of eyes receiving $\mathrm{CL}$ and IOL $(2 \mathrm{~A}=$ treated eye; $2 \mathrm{~B}=$ fellow eye $)$. The means of AL growth of the CL treated and IOL implanted groups were not significantly different $(0.2 \mathrm{~mm}, \mathrm{P}=0.53)$. Table 3 and esupplement figure 3 illustrate $\mathrm{AL}$ in the treated eyes for IOL patients that underwent additional intraocular surgery to clear the visual axis opacification. Axial length growth of eyes receiving surgery to clear the visual axis was significantly more than eyes not receiving surgery for visual axis opacification $(\mathrm{P}=0.013)$. Axial length at age 5 was not significantly different when all eyes were stratified based on visual acuity (table 4 and e-supplement figure 4).

Table 5 (e-supplement reports out of entire cohort. The glaucoma and glaucoma suspect eyes were excluded from the primary analysis but when analyzed as a separate group. Thirteen of 20 glaucoma patients and 11/16 glaucoma suspect patients have AL data in treated eye were 
available for at baseline and at 5 years of age, glaucoma eyes grew more than eyes with glaucoma-suspect or neither (e-supplement table 6).

\section{Discussion}

Prior to cataract surgery, the mean AL was shorter in eyes with cataract as compared to fellow eyes. Similar findings have been reported by other authors. ${ }^{456}$ At age 5 years, the mean AL remained shorter in eyes operated for cataract as compared to fellow unoperated eyes. This result concurs with results published at 1 year of age. ${ }^{2}$ Tadros and colleagues reported similar findings. ${ }^{7}$ In contrast, Vasavada and colleagues reported that mean postoperative AL was longer in eyes with unilateral IOL implantation as compared to fellow unoperated eyes. ${ }^{6}$ The discrepancy in the results may be explained by the fact that in contrast to the series from Vasavada and colleagues, our current study excluded eyes that developed postoperative glaucoma. Glaucoma during early childhood can result in excessive elongation. When we analyzed the excluded eyes separately, the glaucoma eyes did, in fact, grow a mean of $2.4 \mathrm{~mm}$ more than the eyes without glaucoma or glaucoma suspect.

Axial length growth of operative eyes and of fellow unoperated eyes was not significantly different at 5 years of age. This trend was observed at 1 year of age also. ${ }^{2}$ Tadros and colleagues also reported that differences in $\mathrm{AL}$ growth between operated and fellow eyes were not significant ( $4.1 \mathrm{~mm}$ vs $4.4 \mathrm{~mm}, P=0.4$ ). Inamoti and colleagues also reported that there was no significant difference in AL change per year between operated and unoperated eyes. ${ }^{8}$ Flitcroft and colleagues reported no significant difference in AL growth in eyes implanted with an IOL compared to normal eyes. ${ }^{9}$ The authors further reported that the mean of the observed eye growth was not significantly different as compared with the mean of the expected normal value for eye growth. Hussin and Markham ${ }^{10}$ reported similar results for 18 eyes with unilateral congenital cataract after an average follow-up of 9.6 years. In contrast, Greiner and colleagues ${ }^{11}$ reported less AL growth in eyes implanted with an IOL compared with fellow unoperated eyes after a mean of 5.6 years' follow-up in 11 infants with unilateral cataract. Their mean AL growth was $0.46 \mathrm{~mm}$ less in pseudophakic eyes of 7 patients. Leiba and colleagues ${ }^{12}$ showed a tendency toward greater axial lengthening in pseudophakic eyes compared with fellow unoperated eyes after 1 year of follow-up in 9 eyes operated on for unilateral congenital cataract. Kora and colleagues ${ }^{13}$ reported that in 14 unilateral congenital cataract patients, the pseudophakic eyes showed greater AL growth than the fellow unoperated eyes, but their patients were $>2$ years of age at the time of cataract surgery. Vasavada and colleagues ${ }^{6}$ studied the rate of axial growth after congenital cataract surgery at $<1$ year of age in 15 eyes with unilateral cataract and found no significant difference in the axial growth between pseudophakic eyes and fellow eyes after an average of 58.1 months of follow-up.

It is important to note that eyes with cataract were not only shorter as compared to fellow eye without cataract, however, had more variation as reflected by higher standard deviation (Baseline standard deviation 1.2 versus 0.8 in eye with and without cataract; Age 5 standard deviation 1.6 versus 0.7$)$. More variation in eyes operated for cataract was also observed in axial length growth (standard deviation 1.3 versus 0.8 ). 
In the IATS cohort, the mean AL was similar for the cataractous eyes in the two treatment groups when measured just prior to cataract surgery. At 1 year of age, the mean AL of the pseudophakic eyes was $0.6 \mathrm{~mm}$ longer than the mean $\mathrm{AL}$ of the aphakic eyes. ${ }^{1}$ Now, at 5 years of age, the mean AL of the pseudophakic eyes is still longer than the mean AL of the aphakic eyes by $0.5 \mathrm{~mm}$, however this was not statistically significant. Fellow eyes had nearly the same axial elongation in the two treatment groups at 1 year after surgery. Now, at 5 years of age, the fellow eyes had non-significant differences in growth in the two treatment groups.

Prior to cataract surgery, the mean AL was similar for eyes requiring second surgery later on to clear the visual axis. However, at 5 years of age, this trend changed. Eyes requiring surgery to clear the visual axis grew $1.1 \mathrm{~mm}$ more, on average, than eyes without the need for reoperation. Visual axis opacification during infancy can lead to excessive axial elongation if left untreated. Perhaps the duration between the onset of visual axis opacification and surgery to clear the visual axis was sufficient to cause axial elongation from form vision deprivation. In the IATS, patients were examined every 3 months. Therefore, it is unlikely that deprivation from visual axis opacification extended much beyond 3 months in duration. Typically reoperation was scheduled within a few weeks of the recognition of the opacity.

In conclusion, our study showed that eyes operated on for monocular cataract in the first 7 months of life either with or without primary IOL implantation had similar axial growth from surgery until age 5 years to that of fellow unoperated eyes despite having shorter AL at the time of surgery.

\section{Supplementary Material}

Refer to Web version on PubMed Central for supplementary material.

\section{Acknowledgments}

Supported by National Institutes of Health Grants U10 EY13272 and U10 EY013287 and Research to Prevent Blindness, Inc, New York, New York

\section{References}

1. Lambert SR. Changes in Ocular Growth after Pediatric Cataract Surgery. Dev Ophthalmol. 2016; 57:29-39. [PubMed: 27043390]

2. Lambert SR, Lynn MJ, DuBois LG, Cotsonis GA, Hartmann EE, Wilson ME, Infant Aphakia Treatment Study G. Axial elongation following cataract surgery during the first year of life in the infant Aphakia Treatment Study. Invest Ophthalmol Vis Sci. 2012; 53(12):7539-7545. [PubMed: 23074203]

3. Infant Aphakia Treatment Study G. Lambert SR, Buckley EG, Drews-Botsch C, DuBois L, Hartmann E, Lynn MJ, Plager DA, Wilson ME. The infant aphakia treatment study: design and clinical measures at enrollment. Arch Ophthalmol. 2010; 128(1):21-27. [PubMed: 20065212]

4. Kugelberg U, Zetterstrom C, Syren-Nordqvist S. Ocular axial length in children with unilateral congenital cataract. Acta Ophthalmol Scand. 1996; 74(3):220-223. [PubMed: 8828714]

5. Trivedi RH, Wilson ME. Biometry data from caucasian and african-american cataractous pediatric eyes. Invest Ophthalmol Vis Sci. 2007; 48(10):4671-4678. [PubMed: 17898291] 
6. Vasavada AR, Raj SM, Nihalani B. Rate of axial growth after congenital cataract surgery. Am J Ophthalmol. 2004; 138(6):915-924. [PubMed: 15629281]

7. Tadros D, Trivedi RH, Wilson ME, Davidson JD. Ocular axial growth in pseudophakic eyes of patients operated for monocular infantile cataract: a comparison of operated and fellow eyes measured at surgery and 5 or more years later. J AAPOS. 2016; 20(3):210-3. [PubMed: 27109053]

8. Inatomi M, Kora Y, Kinohira Y, Yaguchi S. Long-term follow-up of eye growth in pediatric patients after unilateral cataract surgery with intraocular lens implantation. J AAPOS. 2004; 8(1):50-55. [PubMed: 14970800]

9. Flitcroft DI, Knight-Nanan D, Bowell R, Lanigan B, O'Keefe M. Intraocular lenses in children: changes in axial length, corneal curvature, and refraction. Br J Ophthalmol. 1999; 83(3):265-269. [PubMed: 10365030]

10. Hussin HM, Markham R. Changes in axial length growth after congenital cataract surgery and intraocular lens implantation in children younger than 5 years. J Cataract Refract Surg. 2009; 35(7):1223-1228. [PubMed: 19545812]

11. Griener ED, Dahan E, Lambert SR. Effect of age at time of cataract surgery on subsequent axial length growth in infant eyes. J Cataract Refract Surg. 1999; 25(9):1209-1213. [PubMed: 10476503]

12. Leiba H, Springer A, Pollack A. Ocular axial length changes in pseudophakic children after traumatic and congenital cataract surgery. J AAPOS. 2006; 10(5):460-463. [PubMed: 17070483]

13. Kora Y, Shimizu K, Inatomi M, Fukado Y, Ozawa T. Eye growth after cataract extraction and intraocular lens implantation in children. Ophthalmic Surg. 1993; 24(7):467-475. [PubMed: 8351094] 


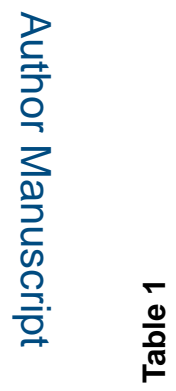

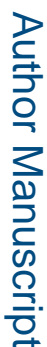

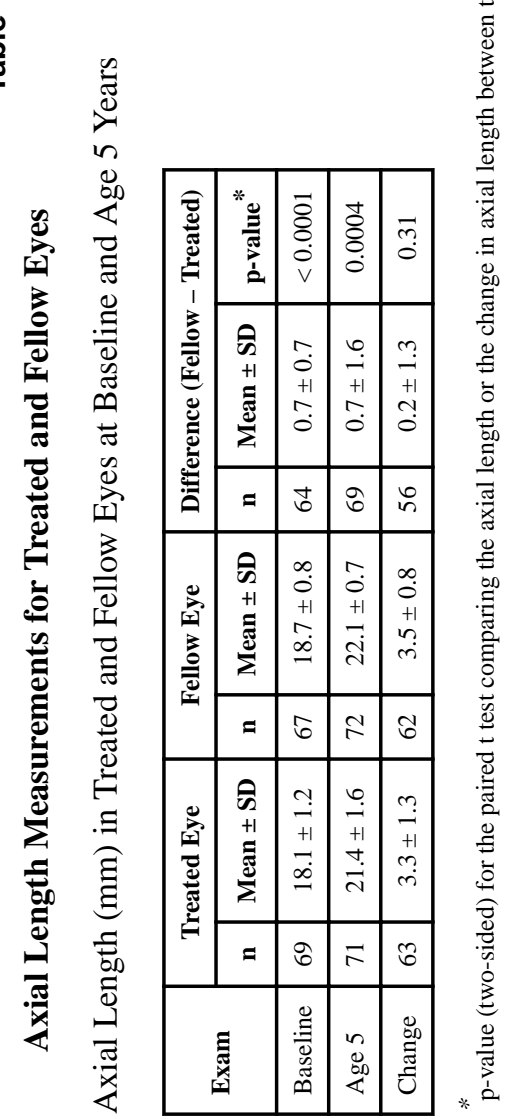

Ophthalmology. Author manuscript; available in PMC 2017 July 16. 


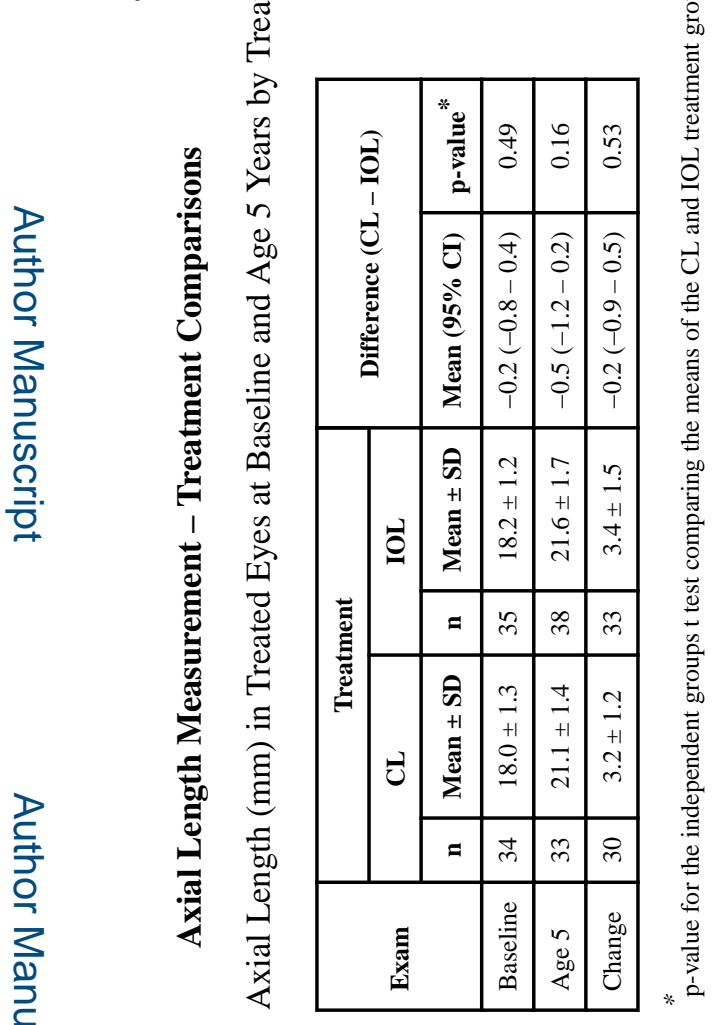


<smiles>[Tl]</smiles>

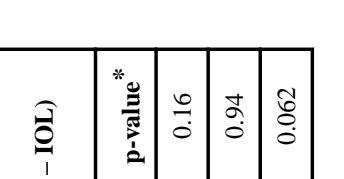

.

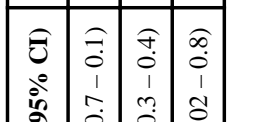




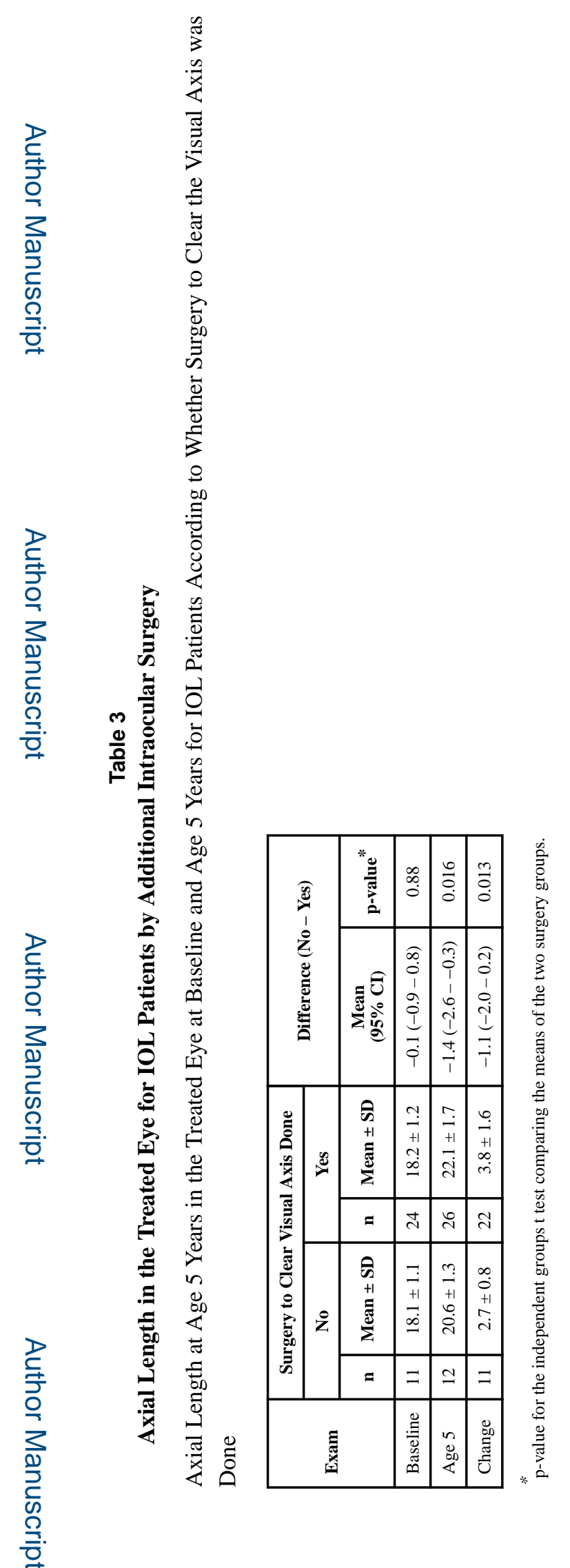

Ophthalmology. Author manuscript; available in PMC 2017 July 16. 


\section{Table 4}

\section{Axial Length and Visual Acuity}

Axial Length at Age 5 Years in the Treated Eye According to Visual Acuity Category at Age 4.5

\begin{tabular}{|l|c|c|}
\hline \multirow{2}{*}{ Visual Acuity Category at Age 4.5 } & \multicolumn{2}{|c|}{ Axial Length at Age 5 (mm) } \\
\cline { 2 - 3 } & N & Mean \pm SD \\
\hline $20 / 20$ to $<20 / 40$ & 11 & $21.1 \pm 1.5$ \\
\hline $20 / 40$ to $<20 / 80$ & 21 & $21.4 \pm 1.6$ \\
\hline $20 / 80$ to $<20 / 200$ & 9 & $21.4 \pm 1.3$ \\
\hline $20 / 200$ or Worse & 29 & $21.5 \pm 1.8$ \\
\hline \multicolumn{2}{|c|}{ p-value $^{*}$} & \multicolumn{2}{|c|}{0.85} \\
\hline
\end{tabular}

p-value for the one-way analysis of variance model comparing the means of the 4 visual acuity categories. 